\title{
LAGUNAS ALTOANDINAS DEL SUR DEL PERÚ: CARACTERÍSTICAS QUIMMICAS
}

Tomás Moreno Beas'

\begin{abstract}
Nueve lagunas y un embalse de reciente construcción han sido estudiados durante el año 1986 en la Cordillera Sur Occidental del Perí con la finalidad de establecer sus caracteristicas químicas y sus variaciones en relación a los periodos de lluvia y sequía. La mayoría de las lagunas se encuentran sobre formación geológica del Altiplano a una altitud que supera los 4300 m.s.n.m., con excepción de una que se halla a 2781 m.s.n.m.
\end{abstract}

Los resultados indican que no obstante encontrarse las lagunas sobre una misma área geológica y bajo las mismas condiciones climáticas, el agua no siempre tiene la misma composición iónica, teniendo respuestas diversas a los cambios de clima. Sus caracteristicas son determinados por la naturaleza química de los constihuyentes de las cuencas, la evaporación y la precipitación de sales en la misma cubeta.

\section{INTRODUCCIÓN}

Según datos de ONERN (1980) el Perú cuenta con unas 12,000 lagunas, muchas de las cuales constituyen importantes recursos para la producción de energía hidroeléctrica, riego agrícola, consumo humano y explotación pesquera. No obstante este enorme potencial, pocos son los estudios de orden limnológico efectuados para establecer las pautas que permitan la explotación racional de éstos recursos.

En la Cuenca del Titicaca existen 841 lagunas, de las cuales existe información mayormente del Lago Titicaca posiblemente por su importancia biogeográfica y la gran extensión de su cuenca; citándose entre los más antiguos, los trabajos de Niethammer (1953), Schindler (1995), Monhein (1956), Gilson (1964), aparte de muchos otros recientes. No existe o es muy escasa la información sobre la gran mayoria de lagunas, con excepción de las publicaciones de Loffler (1968) y Hurlbert et al. (1986).

\footnotetext{
1. Magister en Ecología
}

En este estudio se describen y discuten las características químicas generales y las variaciones entre los períodos de lluvia y sequía, para 8 lagunas de extensión limitada y escasa profundidad, ubicadas sobre la unidad geomorfológica del Altiplano a una altitud superior a los 4300 m.s.n.m.; para un represamiento de reciente construcción sobre la misma altitud y los Andes a los 2781 m.s.n.m.

Quimicamente estas lagunas presentan condiciones muy particulares determinadas por las características geoquimicas propias de sus cuencas, los periodos de lluvia y sequía y la fuerte evaporación; y aunque se encuentran sobre la misma área responden de modo diferente a los cambios de clima. De acuerdo con Loffler $(1968,1972)$ pueden clasificarse como polimicticas frias por su continua circulación a temperaturas que normalmente no exceden los $12-15^{\circ} \mathrm{C}$. Estos cuerpos de agua por su ubicación altitudinal reciben una fuerte radiación solar durante el día que eleva la temperatura en toda la columna sobre los $12^{\circ} \mathrm{C}$; pero en la noche, debido al enfriamiento externo, pierden calor, produciéndose una mezcla total de sus aguas. En épocas frias, la diferencia de la temperatura ambiental entre el día y la noche puede llegar hasta $50^{\circ} \mathrm{C}$ formándose una 
ligera capa de hielo en las zonas de orilla, que se disuelve en horas del medio dia debido a la fuerte y permanente radiación solar.

La mayoria de lagunas objeto de este estudio se encuentran involucradas directa o indirectamente en las obras de afianzamiento de la Laguna Aricota y la construcción del represamiento de Pasto Grande, los cuales tienden a solucionar los problemas energéticos y de carencia de agua para los departamentos de Tacna y Moquegua; por lo que posiblemente las condiciones ecológicas y caracteristicas químicas actuales variarán sustancialmente en el futuro. Estos proyectos amenazan también la existencia de un gran número de aves acuáticas que habitan bofedales y lagunas, asi como las áreas de pastoreo para el ganado auquénido, soporte de las poblaciones humanas asentadas en la zona.

\section{DESCRIPCIÓN DEL ÁREA Y AMBIENTES ESTUDIADOS}

La mayoria de lagunas objeto de este estudio (fig.1) se encuentran sobre el Area Geomorfológica del Altiplano entre los 4300 y 4600 m.s.n.m., con excepción de Laguna Aricota ubicada en la vertiente occidental de la Cordillera de Los Andes a una altitud de 2781 m.s.n.m. (tabla1).

El área se caracteriza por presentar pequeñas planicies conformadas por materiales fluvioglaciares que reciben el nombre de "pampas" (Garcia, 1978) en cuyas depresiones se han formado las lagunas; desarrollándose también áreas pantanosas denominadas localmente "bofedales" (Jaen, 1965), que están constituidas de arenas arcillosas con abundante materia vegetal en descomposición y donde crecen variedad de pastos naturales. Los "bofedales» son un importante hábitat para un gran número de aves acuáticas y lugar de pastoreo de ganado auquénido.

Las lagunas se localizan por lo general entre cerros sobre depósitos Cuaternarios constituidos por cenizas volcánicas, fluvioglaciares y aluviales; entre las que aparecen extrusiones geomorfológicas del Volcánico Barroso y de la Formación Capillune, las cuales son formaciones volcánicas constituidas por tufos y lavas con contenidos de andecitas y piritas (Garcia, 1978).

La geomorfologia de la zona hace suponer que las lagunas que alli se encuentran se han originado como consecuencia de la intensa erosión glaciar del Pleistoceno que conjuntamente produjo
FIGURA N 01: Mapa del Altiplano Occidental del Sur de Perú, donde se muestran las lagunas estudiadas.

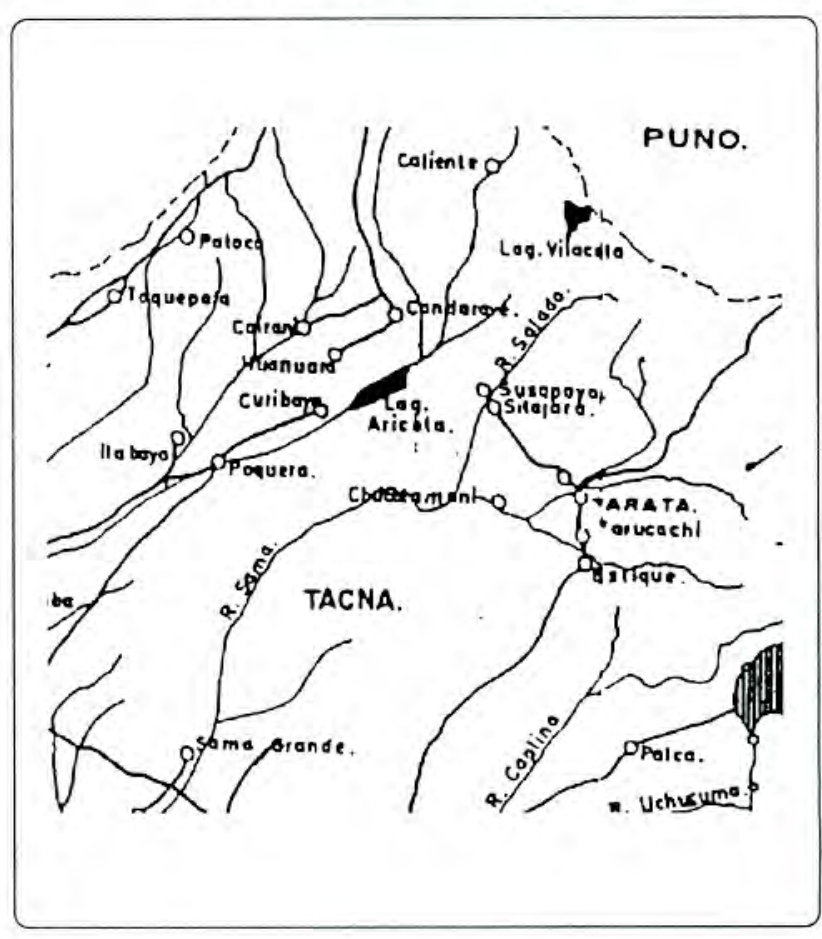

TABLA N 01: Ubicación y características físicas de las lagunas.

\begin{tabular}{|l|l|l|l|l|l|}
\hline \multicolumn{1}{|c|}{ LAGUNA } & LAT. S & LONG. O & $\begin{array}{c}\text { ALTITUD } \\
\text { m.s.n.m. }\end{array}$ & $\begin{array}{c}\text { Z MAX } \\
(\mathrm{m})\end{array}$ & $\begin{array}{l}\text { AREA } \\
(\mathrm{km} 2)\end{array}$ \\
\hline Aricota & $17^{\circ} 22^{\prime}$ & $70^{\circ} 19^{\prime}$ & 2781 & 55 & 14.5 \\
Suches & $16^{\circ} 56^{\prime}$ & $70^{\circ} 24^{\prime}$ & 4450 & 15 & 15 \\
Loriscota & $16^{\circ} 52^{\prime}$ & $70^{\circ} 02^{\prime}$ & 4552 & 4.5 & 33 \\
Vizcachas & $16^{\circ} 53^{\prime}$ & $70^{\circ} 14^{\prime}$ & 4575 & $<2$ & 8.4 \\
Ancocota & $17^{\circ} 09^{\prime}$ & $70^{\circ} 01^{\prime}$ & 4321 & $<1$ & 0.5 \\
Vilacota & $17^{\circ} 08^{\prime}$ & $70^{\circ} 02^{\prime}$ & 4334 & $<0.5$ & 3.3 \\
Rep Jaruma & $17^{\circ} 22^{\prime}$ & $69^{\circ} 57^{\prime}$ & 4475 & $<8$ & 20 \\
Ajuachaya & $16^{\circ} 42^{\prime}$ & $70^{\circ} 13^{\prime}$ & 4560 & $<1$ & 4.6 \\
Caño & $16^{\circ} 53^{\prime}$ & $70^{\circ} 06^{\prime}$ & 4580 & $<0.5$ & 0.8 \\
Loripongo & $16^{\circ} 50^{\prime}$ & $70^{\circ} 05^{\prime}$ & 4555 & $<0.6$ & 0.6 \\
\hline
\end{tabular}

destrucción de conos y rocas volcánicas.

Aricota es una laguna permanente de aguas ligeramente salinas que fue originada por un gran deslizamiento de terreno sobre el cauce del río Curibaya (Jaen, 1965), posiblemente en fecha posterior a la formación de las lagunas ubicadas en la zona altiplánica. Está situada en un valle andino profundo en la vertiente árida occidental y es una de las pocas que se encuentran en alturas intermedias en la vertiente del Pacifico de Los Andes (Scott \& Carbonell, 1986). Años atrás, su profundidad llegaba 
hasta más de $130 \mathrm{~m}$, pero debido a la extracción continua de sus aguas desde 1966 para la provisión de energia hidroeléctrica, en la actualidad sólo tiene $50 \mathrm{~m}$. Se prevee que en corto tiempo se llegará a un nivel critico en que ya no podrá ser explotada, ya que la provisión de agua por sus afluentes, rios Callazas y Salado, no llega a cubrir el volumen que se extrae. Actualmente se ejecutan obras para su afianzamiento con la utilización de recursos hídricos provenientes de la zona Altiplánica. Sus recursos bióticos son muy limitados; han sido observados algunos Anatidae; no se ha confirmado la presencia de peces, aunque existen indicios que existe Salmo gairdnerii.

Laguna Suches se encuentra en una zona fluvioglaciar entre dos flancos del Volcánico Barroso (Garcia, 1978). Es la de mayor profundidad después de Aricota; su forma es casi rectangular y se alimenta de los rios Huaitire y Livecalane y por las filtraciones de los bofedales de la Pampa de Huaitire. De esta laguna nace el río Callazas, el cual acarrea agua sólo en períodos que la laguna alcanza su máximo nivel. $\mathrm{Ha}$ sido construido un dique en su desembocadura con la finalidad de retener el agua para su uso en los centros mineros de Toquepala y Cuajone mediante la extracción con bombas flotantes. Aunque no se halla en peligro de desecación, en períodos de sequía persistente, el nivel de sus aguas baja ostensiblemente. Se han introducido en varias oportunidades alevinos de Salmo gairdnerii que también se crian en jaulas flotantes con resultados satisfactorios. Suches constituye una importante área de nidificación para especies de aves acuáticas altoandinas; se encuentra Podiceps occipitalis y Fulica gigantea, en centenares, así como también Chloephaga melanoptera, Ananas Puna y otras Anatidae. Esporádicamente se han obsenvado Phoenicopterus chilensis.

Laguna Viscacha o Coñocota es una laguna cerrada permanente que se alimenta con aguas del subsuelo y por precipitaciones en época de lluvias. Muestra una turbiedad permanente que imprime un color marrón claro a sus aguas. Han sido introducidos alevinos de Salmo gairdnerii sin resultados satisfactorios. La fauna acuática está conformada por aves con dominancia de Chloephaga melanoptera, encontrándose también varios Anatidae y las tres especies de pariguanas (Phoenicopterus chilensis, $P$. andinatus y Phoenicoparrus jamesi).

Loriscota es la laguna salada de mayor extención en la zona; en época de lluvias se alimenta por los rios Loriza y Putijane y el resto del año por las filtraciones de los bofedales adyacentes. En el periodo de máximo nivel, su profundidad alcanza los $4.5 \mathrm{~m}$, disminuyendo en períodos de sequía. La salinidad de sus aguas tienen su origen en la misma cubeta y se incrementa por evaporación, ya que sus tributarios cargan agua dulce. No obstante su alta salinidad, se proyecta utilizar sus aguas para afianzar la Laguna Aricota. Alberga la mayor cantidad de aves acuáticas de todas las lagunas de la zona, habiéndose registrado en oportunidades hasta 20,000 pariguanas de las tres especies en épocas no reproductivas. También se encuentran Chloephaga melanoptera y otros Anatidae. No existen peces de ninguna especie. La biomasa zooplanctónica, constituida exclusivamente por Boeckella poopoensis es muy abundante.

Laguna Caño se halla ubicada al Sur Oeste de Loriscota en la pampa del mismo nombre. Es una laguna temporal formada por filtraciones y acumulación de agua de bofedales, su profundidad no sobrepasa los $0.5 \mathrm{~m}$ y en épocas de frío congela en sus orillas. Se ha observado abundante número de Fulica gigantea y unas 100 pariguanas (Phoenicopterus chilensis) en el mes de Noviembre. Tiene abundante zooplácton dominado por cladóceros. No tiene peces.

Laguna Loripongo es otra laguna temporal ubicada al Nor-Oeste de Loriscota, formada por filtraciones y agua de bofedales de la Pampa de Ventillo. Su profundidad no sobrepasa los $0.6 \mathrm{~m}$. Han sido observados abundante número de huallatas (Chloephaga melanoptera), chocas (Fulica gigantea), varias especies de Anatidae y en una sola oportunidad se encontraron 11 pariguanas ( $P$. chilensis). EI zooplácton es abundante dominado por cládoceros.

Laguna Vilacota presenta una cubeta amplia de escasa profundidad que se continúa con Laguna Ancocota hacia el Sur-Este. Este grupo de lagunas someras son alimentadas por el rio Quillvire, el cual ha sido canalizado para evitar la pérdida por evaporación y aprovechar sus aguas para afianzar la Laguna Aricota, no existiendo actualmente ingreso de agua a la laguna. La alta evaporación y la carencia de un aporte continuo de agua determinará que en corto tiempo seque totalmente, existiendo el peligro de la pérdida del hábitat para un gran número de aves acuáticas. En mayo de 1986 se ha observado unas 2000 parihuanas de las tres especies, un gran número de Anatidae y Chloephaga melanoptera.

Laguna Ajuachaya o Jaquechía se halla ubicada entre dos cerros, recibe el aporte de la Quebrada Viscallaocco e Istahueco (agua salada) y desemboca en la Pampa de Pasto Grande. Es una 
laguna somera con abundante vegetación en las orillas. Posiblemente en el futuro se modifique el ambiente con la construcción del represamiento de Pasto Grande, el cual cubriria totalmente a la laguna y una gran extensión de tierras aledañas. Han sido observados patos y pariguanas.

La represa de Jaruma se encuentra sobre la cuenca del río Sama en la subcuenca del río Tala (190 km2) y ha sido construida para proveer de agua al Valle de Tarata en épocas de sequía (Ministerio de Agricultura, 1977). El almacenamiento de agua se ha producido recientemente, albergando un volumen de $5.6 \mathrm{mmc}$ a un nivel promedio de $4481 \mathrm{~m}$. s.n.m. Con fines de lograr un total aprovechamiento del recurso, se ha propuesto la introducción de peces autóctonos como la boga (Orestias pentlandii) y el suche (Trichomyterus rivulatus) en forma libre y dispersa, y de la trucha arco iris (Salmo gairdnerii) en corrales o ambientes controlados (Moreno \& Coronel, 1987).

\section{MÉTODOS}

La colección de muestras se realizó en los meses de enero, mayo, agosto, noviembre y diciembre (1986) tomando en consideración los periodos de lluvias y sequias por su importancia en el comportamiento químico de agua.

Para el muestreo, en las lagunas de mayor tamaño y profundidad fue utilizado un bote ligero, estableciéndo dos o más estaciones: en las más someras la colección se efectuó ingresando hasta el lugar que permitía el agua y sedimentos, realizando por lo general una sola estación. Las muestras de agua para los análisis químicos fueron colectadas en frascos plásticos de un litro de capacidad por duplicado. Trasladadas al laboratorio, fueron filtradas con papel Whatman No. 40 y se analizaron de acuerdo a los métodos descritos por Golterman, et al. (1978) y Mackereth, et al. (1978), efectuándose las siguientes determinaciones:

- $\mathrm{pH}$, por medio de un potenciómetro calibrado con soluciones patrón de valores 4.01 y 7.00 a $25 \mathrm{C}$. Las muestras antes de realizar la medición se encontraban a una temperatura constante.

- Conductividad eléctrica, con un conductivímetro YSI Modelo 33 calibrado con cloruro de potasio 0.01 $\mathrm{N}$ a temperatura constante de $25 \mathrm{C}$. Los resultados fueron corregidos a $25 \mathrm{C}$.

- Carbonatos y bicarbonatos por titulación ácida con $\mathrm{HCL} 0.01 \mathrm{~N}$ con indicador de fenolftaleina e indicador mixto.
- Cloruros por titulación con nitrato de plata $0.01 \mathrm{~N} \mathrm{y}$ $0.1 \mathrm{~N}$ de acuerdo con la concentración, usando como indicador cromato de potasio.

- Sulfatos por el método turbimétrico con cloruro de bario, suspendiendo el precipitado en gelatina y realizando las lecturas en el espectrofotómetro a $420 \mathrm{~nm}$.

- Calcio y magnesio se realizaron por complexiometría titulado una muestra con EDTA $0.0100 .1 \mathrm{~N}$, de acuerdo con la concentración.

- Sodio y el potacio se determinaron por fotometría de llama previa calificación del aparato.

- Los sólidos disueltos totales (TDS) se determinaron por evaporación de $50 \mathrm{ml}$ de muestra a $110 \mathrm{C}$, expresándose los resultados en $\mathrm{g} / \mathrm{l}$.

En base a la información reunida se ha calculado el promedio y la desviación standart (Hunt \& Wilsonm 1986) para estableser las condiciones químicas anuales para cada laguna.

\section{RESULTADOS Y DISCUSIÓN}

La concentración representativa promedio y el valor de la desviación standart de los principales iones analizados aparecen en la tabla 2. Las concentraciones se expresan en miliequivalentes por litro para identificar la abundancia relativa de los iones y sus interrelaciones.

\section{CONCENTRACIÓN DE SALES EN SOLUCIÓN}

La concentración salina de las aguas en los lagos estudiados parece depender primariamente del tipo de material rocoso predominante en la cuenca de la evaporación y precipitación (Gibbs, 1970 en Wetzel, 1975) y secundariamente del material transportado por escorrentia. No se descarta la introducción de sales por acción del viento o como producto de la deflación que ocasiona pérdida de sales en lagunas cercanas cuando la aridez es extrema.

La conductividad eléctrica (CE) en Loriscota, laguna sólo con entrada de tributarios y con una gran superficie de evaporación, alcanza más de 15,000 uS cm-1. Vizcachas, que se abastece la mayor parte del año únicamente por aguas del subsuelo tiene valores de CE > 1000 uS $\mathrm{cm}-1$. Las lagunas con entrada y salida continua o que tiene aporte permanente de aguas subterráneas y ubicadas en zonas de escorrentia, muestrar: valores entre 200 y 600 uS cm-1 (Suches, Vilacota, Ancocota, y Ajuachaya). Las lagunas Caño y Loripongo formadas 
por filtraciones y ubicadas en áreas posiblemente no salinas, tienen una CE $<200$ uS cm-1. Laguna Aricota tiene una CE > 1500 uS $\mathrm{cm}-1$ sin duda porque recibe el aporte de ríos que arrastran una cantidad apreciable de sales producto de la erosión y lixiviación de las zonas altas. La represa de Jaruma al estar ubicada en una zona de rocas graníticas y con flujo controlado de entrada y salida de agua tiene una $C E<200$ uS $\mathrm{cm}-1$.

El contenido de sólidos disueltos (TDS) está en relación a la productividad eléctrica. Loriscota tiene $13.42 \mathrm{~g} / \mathrm{l}$ de sólidos en promedio, siguiendo en concentración Aricota y Viscachas con 1.52 y 1.03 respectivamente. El resto de lagunas muestran valores menores de $0.5 \mathrm{~g} / \mathrm{l}$. Loripongo mostró en promedio $0.09 \mathrm{~g} / \mathrm{l}$.

Se ha podido observar que la salinidad muestra una variabilidad marcada en relación con los períodos de lluvia y sequía pero no existe un mismo comportamiento en todas las lagunas. En Aricota en la época de sequia (Agosto), la conductividad eléctrica es más alta en los meses de lluvia (noviembrediciembre) y disminuye, mostrando un valor intermedio en el més de mayo, periodo de finalización de lluvias. En Suches en cambio, la conductividad eléctrica en los meses de lluvia podría deberse al ingreso de grandes cantidades de agua de baja salinidad como producto de las precipitaciones.

En las lagunas Loriscota y Viscachas, se observa que en los meses húmedos la $C E$ es ligeramente más baja que en los meses de sequia sin duda por el ingreso de aguas de baja salinidad; este hecho, permite indicar que el aumento de la concentración salina en épocas de sequia es gobernada por la evapotranspiración que alcanza una media anual de $285.1 \mathrm{~mm}$, con relación a una precipitación media anual de $417.2 \mathrm{~mm}$ (Vera, 1988).

Las lagunas cerradas que son alimentadas por aguas del subsuelo y por acumulación de precipitaciones pluviales, muestran una menor concentración de sales en los meses de lluvia, que va aumentando conforme se incrementa la aridez; tal es el caso de Lagunas Caño y Loripongo.

Laguna Ancocota es una cubeta abierta con una influencia muy marcada de la escorrentía y puede notarse que existe una gran diferencia en el contenido salino entre los meses de lluvia y sequía. Los datos no son suficientes para apreciar las variaciones de la salinidad con los periodos de lluvia y sequia en todas las lagunas, pero la distribución mensual promedio de la escorrentía, nos da una posible indicación de las variaciones que ocurririan. Datos de Vera (1988) indican un promedio de la escorrientía de 16.60 de marzo a mayo y de 18.80 de junio a agosto.

pH : El pH en las lagunas cerradas y en aquellas que tienen poca provisión de agua es fuertemente alcalino con valores $>8.3 y<9.6$, posiblemente debido a la presencia de alcalino térreos y sedimentos calcáreos; en las lagunas abiertas con un volumen grande de agua el pH varía entre 7.0 y 7.39 (Aricota, Suches y Jaruma). La única laguna con $\mathrm{pH}$ ácido (3.4) y de aguas sulfatadas es Ajuachaya, fenómeno que podria explicarse porque se encuentra sobre un área abundante en sulfuro de fierro (pirita) y tiene influencia de aguas volcánicas.

\section{CONCENTRACIÓN DE IONES}

La proporcionalidad en la concentración de aniones es muy diversa, encontrándose los siguientes tipos de aguas:

$\begin{array}{ll}\text { Tipo bicarbonato } & \\ \mathrm{HCO} 3>\mathrm{SO} 4>\mathrm{CL} & \text { Rep. Jaruma y Caño } \\ \mathrm{HCO} 3>\mathrm{CL}>\mathrm{SO} 4 & \text { Loripongo y Vizcachas } \\ \text { Tipo sulfato } & \\ \mathrm{SO} 4>\mathrm{HCO} 3>\mathrm{Cl} & \text { Suches y Vilacota-Ancocota } \\ \mathrm{SO} 4>\mathrm{Cl}>\mathrm{HCO} 3 & \text { Ajuachaya } \\ \text { Tipo cloruro } & \\ \mathrm{Cl}>\mathrm{SO} 4>\mathrm{HCO} 3 & \text { Aricota } \\ \mathrm{Cl}>\mathrm{HCO} 3>\mathrm{SO} 4 & \text { Loriscota }\end{array}$

En el primer tipo los carbonatos, presentes como bicarbonatos, exceden a los sulfatos y a los cloruros; característica de aguas duras donde deben estar presentes sedimentos calcáreos (Hutchinson, 1975). El tipo sulfato se da por lo general por la influencia de aguas volcánicas o por la solubilidad de sulfatos, como ocurre en las orillas de laguna Suches, donde existe un depósito de azufre. El último tipo es característico de lagunas donde predomina el sodio.

Las proporciones iónicas de los cationes son relativamente uniformes y muestran relación con las formaciones rocosas, encontrándose las siguientes relaciones:

$$
\begin{array}{ll}
\mathrm{Na}>\mathrm{Ca}>\mathrm{Mg}>\mathrm{K} & \begin{array}{l}
\text { Aricota, Suches, Vilacota-Ancocota, } \\
\text { Ajuachaya, Caño y Loripongo. }
\end{array} \\
\mathrm{Na}>\mathrm{K}>\mathrm{Ca}>\mathrm{Mg} & \text { Loriscota y Viscacha } \\
\mathrm{Ca}>\mathrm{Na}>\mathrm{Mg}>\mathrm{K} & \text { Rep. Jaruma }
\end{array}
$$

La mayoría de lagunas muestran un patrón de concentración del tipo $\mathrm{Na}>\mathrm{Ca}>\mathrm{Mg}>\mathrm{K}$, que reflejaria la presencia de tierras alcaninas y 
sedimentos calcáreos. La dominancia del ión sodio junto con el cloruro posiblemente se debe a fuentes salinas o lixiviación de sedimentos salinos en la cubeta, aunque existe también la posible intervención de un proceso químico en que los bicarbonatos y el calcio formen precipitados de $\mathrm{CaCO} 3$ con liberación de dióxido de carbono (Ambe, 1984), determinando que el sodio aparezca en mayor concentración. Este proceso se llevaría a cabo de acuerdo a las siguientes reacciones:

$\mathrm{Na}++\mathrm{Ca}+++2 \mathrm{HCO}_{-}-=\mathrm{Na}++\mathrm{CaCO} 3 \downarrow+\mathrm{CO}_{2} \uparrow+\mathrm{H}_{2} \mathrm{O}$

Hutchinson (1975) indica que en tierras alcalinas, cuando la concentración de bicarbonatos y álcalis es alta, la evaporación precipita el calcio como CaCO3; si todavía existe calcio en solución, arrastraria también al sulfato en forma de CaSO4.2H2O. Como resultado, las aguas tendrian como mayores constituyentes bicarbonato de sodio y carbonatos, junto al cloruro como principal anión. Este fenómeno estaría ocurriendo en Loriscota, donde se ha observado acumulaciones de carbonato de calcio y la concentración de sodio y cloruro es muy alta.

El sodio por lo general se presenta en mayor concentración que el calcio y magnesio en la mayoria de lagunas, con excepción de la Represa de Jaruma que muestra un valor intermedio entre los dos cationes. La mayor concentración de sodio se ha registrado en Loriscota ( $160.12 \mathrm{meq} / \mathrm{l})$ como producto de la disolución de sales de la misma cubeta y la precipitación del calcio en forma de carbonatos, ya que los tributarios ríos Lorisa y Putijane no arrastran cantidades significativas de sodio. Este mismo fenómeno también se produciria en Viscachas donde el contenido de sodio es alto. En Aricota, a diferencia de las anteriores, se ha establecido que el sodio ingresa por aporte de los ríos Salado y Callazas, siendo su concentración mayor que otros cationes.

El potasio aparece en segundo nivel de importancia en Viscachas y Loriscota, posiblemente debido al intercambio catiónico con algunas arcillas del tipo de la illita, ya que las proporciones de los cationes se modifican por cambio con las arcillas, la

\section{REFERENCIAS BIBLIOGRÁFICAS}

GARCIA MARQUEZ, W. 1978. Geologia de los Cuadrángulos de Puquina, Omate, Huaitire, Mazocruz y Pizacoma. Instituto de Geología y Mineria. 63pp.

HUNT, D.T.E. \& A.L. WILSON 1986. The Chemical Analysis of Water: General Principles and Techniques. Second Edition. The Royal Society of Chemistry. London. 683 pp. materia orgánica y el suelo. Las arcillas normalmente retienen más los cationes monovalentes que los divalentes, de modo que el calcio y magnesio se movilizarian con más libertad; pero en condiciones de exceso de sodio, la reserva alcalina está constituida no sólo por alcalino térreos, sino también por alcalinos, dando lugar a aguas carbonatadas-sódicas en las que precipita el calcio, de modo que la proporción entre la suma de sodio y potasio y la suma de cloruro y sulfatos aparece de este modo: $\mathrm{Na}+\mathrm{K}>\mathrm{Cl}+\mathrm{SO} 4$ (Margalef, 1983). Este tipo de proporción es encontrada en las lagunas Loriscota, Viscachas, Caño y Lorispongo.

Los datos permiten establecer que el agua de los lagos con conductividad eléctrica $>1000$ uS $\mathrm{cm}-1$ tienen dominancia de sodio y cloruros y en algunos casos sodio y bicarbonatos (Vizcachas); en aguas cuya conductividad varía entre 200 y 600 uS $\mathrm{cm}-1$ existe dominancia de sodio y sulfatos y aguas con valores de conductividad menores de $200 \mathrm{uS} \mathrm{cm}$ 1 muestra una dominancia de sodio y bicarbonatos 0 calcio y bicarbonatos.

No es posible establecer la variación estacional en las concentraciones iónicas debido a las mezclas que se producen entre ellos, principalmente en los períodos de lluvias. Pero se observa que algunos aniones como los carbonatos y sulfatos mantienen casi su mismo nivel de concentración durante todo el año y similar comportamiento tienen el potasio y el magnesio.

Los resultados de este estudio indican que las lagunas que se encuentran en la misma área geográfica no necesariamente tienen la misma composición iónica y no responden idénticamente a los cambios de clima. Son la naturaleza geoquímica de los afluentes y la interacción e intercambio de iones entre agua y los sedimentos de los fenómenos que determinan las propiedades quimicas del agua en las lagunas abiertas. En las lagunas cerradas, aparte de las características geoquímicas de las cubiertas, juegan un rol importante la evapotranspiración y precipitación química.

HURLBERT, S.H.; W. LOAYZA AND T. MORENO. 1986. FishFlamingo-Planckton Interactions in the Peruvian Andes. Limnol. Oceanogr. 31(3):457-468.

HUTCHINSON, G.E. 1975. A Treatise on Limnology. Volume I, Part 2: Chemistry of Lakes. Second Edition. Jhon Wiley \& Sons. New York. pag. 552-574. 
JAEN DE LA TORRE, H. 1965. Geologia del Cuadrángulo de Tarata. Comisión Carta Geológica Nacional. 91 pp

MARGALEF, R. 1983. Limnologia. Edición Omega, S.A. Barcelona. $1010 \mathrm{pp}$

MINISTERIO DE AGRICULTURA. 1977. Represamiento de Jaruma. Actualización Hidrológica. Dirección de estudios: Estudio Integral de las cuencas de Tacna y Moquegua. $71 \mathrm{pp}$.

MONHEIM, F. 1956. Bietrage zur Klimatologie und Hydrologie des Titicacabeckens. Heidelberger Geogr. Arb. 1:1152.
MORENO, T. Y N. CORONEL. 1987. Estudio Posibilidad de Introducción de Especies No Nativas. Conveni UNJBG-Micro Región TARata. $82 \mathrm{pp}$.

ONERN.1980. Inventario Nacional de Lagunas y Represamientos (Segunda Edición) Oficina Nacional de Evaluación de Recursos Naturales. Lima.

VERA, G. 1988. Estudio Hidrológico de las Cuencas del Proyecto Kovire. Ministerio de la Presidencia, Instituto Nacional de Desarrollo

TABLA No. 2: Parámetros quimicos representativos de las lagunas de la zona altiplánica occidental dei Sur del Perú.

\begin{tabular}{|c|c|c|c|c|c|}
\hline \multirow{2}{*}{\multicolumn{2}{|c|}{$\begin{array}{c}\text { LAGUNA } \\
\text { ESTADISTICO } \\
(\mathrm{n})\end{array}$}} & \multicolumn{2}{|c|}{ ARICOTA } & \multicolumn{2}{|c|}{ SUCHES } \\
\hline & & MEDIA & 10 STD & MEDIA & STD \\
\hline $\mathrm{pH}$ & $25 \mathrm{C}$ & 7.23 & 2.42 & 7.26 & 0.30 \\
\hline uS $\mathrm{cm}-1$ & $25 \mathrm{C}$ & 1668.59 & 285.69 & 206.35 & 30.86 \\
\hline HCD3- & $\mathrm{meg} / \mathrm{l}$ & 2.92 & 0.99 & 0.95 & 0.18 \\
\hline CO3-- & $\mathrm{meg} / \mathrm{l}$ & 0.24 & 0.33 & 0.00 & 0.00 \\
\hline C1- & $\mathrm{meg} / \mathrm{l}$ & 9.85 & 0.39 & 0.34 & 0.12 \\
\hline SO4-- & $\mathrm{meg} / \mathrm{l}$ & 5.92 & 0.46 & 1.11 & 0.25 \\
\hline SUMA DE & ANTONES & 18.92 & 1.06 & 2.40 & 0.33 \\
\hline $\mathrm{Ca}+$ & $\mathrm{meg} / \mathrm{l}$ & 5.11 & 0.37 & 0.83 & 0.21 \\
\hline $\mathrm{Mg}^{+}$ & $\mathrm{meg} / 1$ & 3.33 & 0.34 & 0.76 & 0.26 \\
\hline $\mathrm{Na}+$ & $\mathrm{meg} / \mathrm{l}$ & 10.51 & 1.34 & 0.87 & 0.11 \\
\hline $\mathrm{K}+$ & $\mathrm{meg} / \mathrm{l}$ & 0.72 & 0.06 & 0.15 & 0.01 \\
\hline SUMA DE & CATIONES & 19.67 & 1.59 & 2.60 & 0.37 \\
\hline T.D.S. & $G / I$ & 1.52 & 0.38 & 0.16 & 0.08 \\
\hline \multicolumn{2}{|l|}{ LAGUNA } & \multicolumn{2}{|c|}{ VILACOTA } & \multicolumn{2}{|c|}{ JARUMA } \\
\hline \multicolumn{2}{|c|}{$\begin{array}{c}\text { ESTADISTICO } \\
(n)\end{array}$} & \multicolumn{2}{|c|}{3} & 8 & STD \\
\hline $\mathrm{pH}$ & $25 \mathrm{C}$ & 8.63 & 1.07 & 7.01 & 0.47 \\
\hline uS $\mathrm{cm}-1$ & $25 \mathrm{C}$ & 312.78 & 106.08 & 189.99 & 3.76 \\
\hline HCD3- & $\mathrm{meg} / \mathrm{l}$ & 1.01 & 0.32 & 1.54 & 0.15 \\
\hline CO3-- & $\mathrm{meg} / \mathrm{l}$ & 0.49 & 0.47 & 0.04 & 0.11 \\
\hline C1- & $\mathrm{meg} / \mathrm{l}$ & 0.29 & 0.06 & 0.43 & 0.18 \\
\hline SO4-- & $\mathrm{meg} / \mathrm{l}$ & 2.12 & 1.22 & 0.48 & 0.08 \\
\hline SUMA DE & ANTONES & 3.91 & 1.42 & 2.49 & 0.16 \\
\hline $\mathrm{Ca}+$ & $\mathrm{meg} / \mathrm{l}$ & 1.21 & 0.45 & 1.07 & 0.30 \\
\hline $\mathrm{Mg}^{+}$ & meg/l & 0.72 & 0.27 & 0.68 & 0.03 \\
\hline $\mathrm{Na}+$ & meg/l & 1.86 & 0.91 & 0.93 & 0.01 \\
\hline $\mathrm{K}+$ & $\mathrm{meg} / \mathrm{l}$ & 0.30 & 0.12 & 0.11 & 0.00 \\
\hline SUMA DE & CATIONES & 4.10 & 1.44 & 2.79 & 0.30 \\
\hline T.D.S. & $\mathrm{G} / \mathrm{I}$ & 0.23 & 0.04 & 0.13 & 0.03 \\
\hline
\end{tabular}

\begin{tabular}{|r|r|r|r|r|r|}
\hline \multicolumn{2}{|c|}{ LORISCOTA } & \multicolumn{2}{c|}{ VIZCACHAS } & \multicolumn{2}{c|}{ ANCOCOTA } \\
\hline \multicolumn{1}{|r|}{ MEDIA } & STD & \multicolumn{1}{c|}{ MEDIA } & STD & MEDIA & STD \\
\hline 9.55 & 0.48 & 8.33 & 0.22 & 9.25 & 0.84 \\
15628.60 & 1948.80 & 1138.66 & 191.37 & 628.64 & 212.10 \\
& & & & & \\
25.04 & 6.94 & 6.46 & 1.57 & 1.17 & 0.83 \\
16.77 & 1.97 & 0.48 & 0.54 & 0.79 & 0.43 \\
134.96 & 1.63 & 3.25 & 0.50 & 0.64 & 0.18 \\
2.77 & 0.89 & 2.20 & 0.72 & 2.58 & 1.48 \\
179.53 & 13.80 & 12.39 & 2.69 & 5.18 & 1.07 \\
& & & & & \\
0.43 & 0.12 & 0.95 & 0.28 & 1.84 & 0.67 \\
0.27 & 0.11 & 0.76 & 0.18 & 0.52 & 0.13 \\
160.12 & 18.20 & 8.58 & 2.38 & 3.56 & 1.09 \\
13.60 & 1.43 & 3.13 & 0.39 & 0.54 & 0.14 \\
174.42 & 18.13 & 13.42 & 2.71 & 6.46 & 1.94 \\
13.42 & 2.75 & 1.03 & 0.34 & 0.48 & 0.17 \\
\hline
\end{tabular}

\begin{tabular}{|r|r|r|r|r|r|}
\hline \multicolumn{2}{|c|}{ AJUACHAYA } & \multicolumn{2}{c|}{ CAÑO } & \multicolumn{2}{c|}{ LORIPONGO } \\
\hline MEDIA & STD & MEDIA & STD & MEDIA & STD \\
\hline 2 & & \multicolumn{2}{|c|}{4} & \multicolumn{2}{c|}{3} \\
\hline 3.40 & 0.10 & 8.80 & 0.74 & 9.23 & 0.47 \\
315.60 & 82.08 & 185.16 & 37.02 & 166.23 & 18.13 \\
& & & & & \\
0.00 & 0.00 & 1.07 & 0.71 & 0.75 & 0.16 \\
0.00 & 0.00 & 0.57 & 0.39 & 0.69 & 0.17 \\
0.34 & 0.03 & 0.37 & 0.13 & 0.56 & 0.15 \\
2.72 & 0.59 & 0.40 & 0.56 & 0.13 & 0.04 \\
3.06 & 0.56 & 2.42 & 0.68 & 2.14 & 0.44 \\
0.47 & 0.07 & 0.49 & 0.16 & 0.67 & 0.20 \\
0.39 & 0.08 & 0.35 & 0.01 & 0.45 & 0.08 \\
0.66 & 0.05 & 1.14 & 0.23 & 0.83 & 0.07 \\
0.10 & 0.03 & 0.32 & 0.08 & 0.24 & 0.02 \\
1.60 & 0.23 & 2.30 & 0.42 & 2.20 & 0.21 \\
0.17 & 0.15 & 0.19 & 0.09 & 0.09 & 0.07 \\
\hline
\end{tabular}

